Voix et Images

volxetimages

\title{
Les corps scellés. Analyse de trois romans de Monique Bosco
}

\section{François Gallays}

Volume 9, numéro 3, printemps 1984

Monique Bosco

URI : https://id.erudit.org/iderudit/200478ar

DOI : https://doi.org/10.7202/200478ar

Aller au sommaire du numéro

\section{Éditeur(s)}

Université du Québec à Montréal

\section{ISSN}

0318-9201 (imprimé)

1705-933X (numérique)

Découvrir la revue

\section{Citer cet article}

Gallays, F. (1984). Les corps scellés. Analyse de trois romans de Monique Bosco. Voix et Images, 9(3), 35-45. https://doi.org/10.7202/200478ar d'utilisation que vous pouvez consulter en ligne.

https://apropos.erudit.org/fr/usagers/politique-dutilisation/ 


\title{
Les corps scellés. Analyse de trois romans de Monique Bosco
}

\author{
par François Gallays, Université d'Ottawa
}

La première fois que je lus le titre Un amour maladroit', l'adjectif a fonctionné spontanément comme le second terme d'une métaphore, mais au moment où j'ai achevé la lecture du texte du roman, s'est imposé rétrospectivement son sens littéral. En effet, au premier contact avec le titre, obéissant à une certaine habitude de lecture ou à l'habitude de lecture d'une certaine littérature, le lecteur confère à la maladresse de cet amour dont il est question une valeur spirituelle, y voit l'expression d'une certaine discordance au niveau des rapports affectifs alors que, le roman terminé, le même lecteur se rend compte que la maladresse avant que d'être de nature spirituelle ou affective fut d'abord et avant tout de nature physique. Car l'échec amoureux auquel renvoie le titre ne constitue pas autre chose que la dernière séquence de l'histoire d'un corps désaccordé, n'est que l'aboutissement en d'autres mots de l'apprentissage raté d'un corps comme objet social, que le dernier acte de sa socialisation carencée.

Roman d'apprentissage donc, comme le sont souvent les premiers romans, Un amour maladroit emprunte la forme du récit auto-diégétique pour mettre en scène Rachel, jeune femme dans la vingtaine qui, ayant émigré vers le Canada à l'invitation de sa tante Marthe, au veuvage récent, connaît une adaptation difficile et accepte, après encouragement de la part de sa tante, de suivre un cure psychanalytique. Elle se trouve néanmoins un emploi dans un journal de la métropole pour tomber, quelques mois plus tard, amoureuse d'un journaliste, genre Rastignac, qui, lui, n'aime que sa propre personne.

Autre lieu commun, le roman s'ouvre sur la déclaration justificatrice de la narratrice: le récit qu'elle entreprend de son passé a pour but de reprendre en main sa propre vie, d'atteindre la vérité sur elle-même au-delà 
de la multiplicité de ses masques afin aussi d' «apprendre à connaître les autres ${ }^{2} »$. Ainsi, d'une certaine façon, le lecteur est d'emblée, par rapport au récit qui s'amorce, mis en position d'écoute. Comme l'analyste, le lecteur assistera, témoin muet, à la narration des souvenirs de la jeune femme. Le rapprochement entre la forme du texte et la forme rhétorique de l'écoute n'est pas pure gratuité car, outre ce début du récit qui le suggère, la fin le suggère encore plus fortement. Dans le chapitre pénultième du texte, qu'il est légitime d'envisager comme le chapitre clausulaire alors que le dernier chapitre fait quasiment figure de note surajouté, donc dans ce chapitre pénultième, surgit la figure d'un narrataire, dont l'identité n'est pas immédiatement reconnue. Quelle est l'identité précise du narrataire, se demande le lecteur? Est-ce moi, lecteur? Est-ce le psychanalyste? Ou sont-ce les deux simultanément? Il faut attendre la troisième phrase du chapitre pour que soit levée l'ambiguîté:

L'un devant l'autre, à nouveau, comme pour cette première confrontation où je vous ai livré ma vie, tel un livre ouvert dont je n'aurais point su déchiffrer le contenu. Merci de cette confiance. Après le divan psychanalytique ${ }^{3}(. .$.$) .$

La comparaison "tel un livre ouvert», loin de dissiper la confusion momentanée entourant l'identité du narrataire, la maintient en encourageant un certain brouillage entre le texte écrit et le «texte» de l'écoute. Dans cette perspective, la cure que poursuit Rachel à Montréal et dont la fin coïncide avec celle du roman fonctionne comme une mise en abyme du récit. Miroir à l'intérieur d'un autre miroir car, dès les premières lignes du texte, la narratrice présente le récit comme le miroir qu'elle dressera devant son visage pour enfin se connaître. Mais ce faisant, détail significatif, la narratrice reprend pour son compte, en les pastichant, les célèbres paroles prononcées par la mauvaise reine dans le conte de Blanche-Neige: «Miroir, dismoi qui je suis: ni la plus belle ni la plus laide, mais je ne puis supporter davantage d'être une inconnue à mes propres yeux ${ }^{4} . »$ Ce détour intertextuel révèle assez clairement que la connaissance de soi passera d'abord par la reconnaissance et par l'acceptation de son propre corps («.. ni la plus belle, ni la plus laide...»).

Très tôt, et pendant longtemps, depuis les premiers souvenirs, la narratrice n'eut d'autre identité auprès des siens que le trait dominant de son corps: la Petite. Et, symbolisme un peu trop facile il me semble, son nom véritable, Rachel, n'est dévoilé au lecteur qu'à la fin de la cure. Plus tard, toujours affublée de son seul surnom, inversant les rôles traditionnels, c'est en petite soeur que Rachel se comportera auprès d'Élisabeth sa cadette de deux ans qui, à treize ans, «avait déjà une allure de jeune femme», alors que Rachel affichait encore, hélas! une «silhouette informe, où les rondeurs tant escomptées ne voulaient pas se décider à faire leur apparition".» Mais auparavant, Rachel, d'origine polonaise, émigrée en France, vit, dès son 
premier jour a l'école, son corps malingre, recouvert d'étoffes flamboyantes à la mode de son pays d'origine, tourné en ridicule par les petites Françaises dont les corps, enveloppés dans d'affligeantes blouses noires, affichaient la superbe toute cléricale de l'école républicaine.

Trahie sur tous les plans par son corps disgracieux (les périodes d'exercices physiques laisseront d'atroces souvenirs), Rachel se mit à le honnir vraiment le jour où elle apprit qu'il avait aux yeux de son oncle une allure sémitique. Réfugiée à Marseille pour échapper à la terreur nazie qui sévissait à Paris, sa famille, c'est-à-dire sa mère, sa soeur, sa grand-mère, sa tante et son oncle, vit coincée dans un minuscule appartement. Le filet nazi se resserrant, l'oncle, redoublant de prudence, interdit à Rachel et à sa grandmère de jamais sortir de l'appartement, parce que, décréta-il, parmi les membres de la famille, ce sont elles qui avaient l'air le plus juif:

"Je te hais», disais-je à mon reflet. Il me semblait d'une injustice suprême d'avoir à porter au grand jour, au clair le secret de mon identité. Je m'appliquais à me décerner le plus grand nombre possible de «signes distinctifs». Je m'amusais à détester à tour de rôle, mes yeux, mes mains, mon teint. «Tu es laide et bête.) Dans la glace, mon reflet avait maintenant l'intensité mauvaise que l'on voit aux repris de justice sur les fiches anthropométriques $^{6}$.

Mais à la «trahison» de son corps, à partir de laquelle se développe chez Rachel une sorte de narcissisme à l'envers qu'on ne saurait qualifier autrement que de morbide, s'ajoute une «trahison» peut-être plus fondamentale encore, irréductible en tout cas, la trahison parentale: ce père fantasmé et désiré d'autant plus intensément qu'il abandonna le foyer avant la cristallisation de tout souvenir. Et cette mère, belle, mais froide, mais distante qui jamais ne prodigua à ce corps assoiffé de petite fille la moindre caresse. Entre le corps malingre, disgracieux et doublement mal-aimé de l'adolescente et le corps amoureux maladroit de jeune femme, le texte du roman établit, par l'entremise d'analepses fréquentes, une relation directe de causalité. Cependant, le corps amoureux comme tel est censuré dans le texte. Et ceci est sans doute en partie attribuable à la convention romanesque de l'époque, mais les traces du comportement du corps de Rachel durant les tentatives de fréquentations' qui précèdent l'expérience amoureuse révèlent un corps socialement si démuni, aux inhibitions si paralysantes qu'il est impossible de ne pas envisager cette censure comme le refoulé du texte, comme le texte essentiel autrement dit, mais tu. De plus, la haine du corps demeure vivace: 
et la rancune anciennes demeuraient. Désormais, en ces années de vaches grasses, je me plaisais à lui refuser le superflu comme le nécessaire. À le voir s'étioler, s'amenuiser, se réduire à rien, mon sens de la justice était tardivement satisfait. Gourmand, exigeant, réclamant à cor et à cri sa portion quotidienne, je n'avais entendu que lui au cours de ces années de restrictions et de famine. $\grave{A}$ le voir si maigre et décharné, réduit à la peau et aux os, une sorte de pitié me saisissait parfois; ou bien encore je me réjouissais: "Bien fait pour toi.» N'était-il pas juste qu'il souffre à son tour, qu'il succombe sous le poids de ses fautes: «Bon à rien, maladroit, incapable, lourdeau ${ }^{8} . »$

Avec un corps si mal aimé, avec un corps si méprisé, l'échec amoureux était inévitable. D'autant plus qu'aux yeux de Rachel, cet amour lui parut, du moins rétrospectivement, posséder toutes les qualités salvatrices nécessaires pour enfin l'arracher à son marasme intérieur dans lequel depuis son enfance elle semblait s'enfoncer de plus en plus profondément. De telles exigences ne purent que grever sévèrement sa relation avec Yves Dumont et ce, dès le début. Aussi ne se développa-t-elle jamais vraiment. Loin de la lumière, comme si elle fuyait le regard public, leur liaison se limita au furtives visites dans les «chambres morbides» d'hôtels de passe. Tristesse. Aussi y mit-elle fin (coïncidence un peu facile) au moment où se modifia son attitude envers son psychanalyste. Ces deux événements précèdent de peu la résolution de n'offrir plus prise au monde. Le corps, dont l'ouverture vers l'autre et vers le monde fut fugitivement entrevue le temps de cet amour malheureux, colmate la brèche et se referme sur lui-même:

Me voilà enfin fermée, dure et méchante, désireuse de rendre le mal pour le mal, de faire souffrir à mon tour, de me venger. (...)

J'ai refermé la porte sur mon passage. Me voilà calfeutrée, inatteignable, en l'abri que je me suis creusée. (...)

J'ai besoin (...) de ce goût de la mort en moi. J'ai tant lutté pour préserver en moi cette part obscure, cette région d'ombre, ignorée. Là, lentement, à l'abri de tous et surtout de ma conscience, j'édifiais le mur qui me séparait à jamais des autres. (...) Derrière son enceinte plus rien ne pourra jamais m'atteindre, ni en bien ni en mal'. 
qui sépare le corps de Rachel du monde, le texte fait appel de nouveau aux images de la pierre et du désert pour évoquer la stérilité dont est frappé ce corps:

Pierre et sable. Rien ne pousse ni ne survit en cette aridité. Me voilà morte déjà, et contente de l'être ${ }^{10}$.

À ce chapitre huit, aux propos extrêmes par l'entremise desquels s'expriment le froid désespoir de Rachel et la condamnation de son corps a l'autisme, d'où il n'avait jamais réussi tout à fait à s'extraire, succède le dernier chapitre, genre de post-scriptum, où Rachel en quelques lignes résume de grands pans de sa vie faite de solitude, de travail et de résignation. En d'autres mots, le texte s'achève sur une sorte de médiocrité assumée, alors que le chapitre précédent s'achevait sur la désolation et la mort.

Ả la fin d'Un amour maladroit (chapitre 8), il est une phrase à rappeler, car par son entremise se tissent des liens étroits avec le troisième roman de Bosco, La Femme de Loth:

J'aime cette insidieuse maladie qui me transforme lentement, inexorablement, en une statue de sel ${ }^{11}$.

Le pont que cette allusion au destin de la femme de Loth établit entre les premier et troisième romans de Monique Bosco ne signale pas seulement une simple filiation, car la Femme de Loth ${ }^{12}$, à la fois par sa forme et son contenu, se laisse assez aisément réduire à une sorte de «remake» d'Un amour maladroit.

Hélène, une femme de quarante ans, après avoir vécu une liaison clandestine de dix ans avec un homme du nom de Pierre, marié et père de famille, se retrouve soudain seule, profondément blessée et complètement désemparée, car son amant, sans ménagement, a mis fin à leur commune expérience amoureuse:

Dix ans, j'ai sagement accepté mon sort. Au seuil de la terre promise, à l'ombre du grand homme adoré. Sans un murmure. Esclave reconnaissante. J'ai trahi le commandement, loi impitoyable interdisant de faire des dieux à effigie humaine ${ }^{13}$.

Le lendemain, pour voir clair, mais aussi, et surtout, pour échapper au couperet de la vérité ( "Je n'écris ce roman que pour éloigner l'échéance de la vérité $\left.{ }^{14} . »\right)$, Hélène s'attable devant son paquet de feuilles blanches et se met à fabriquer un roman, dont elle sera le personnage central. (Rachel 
n'est jamais loin.) Installée ensuite à Venise, elle y terminera son «oeuvre». On le voit, la Femme de Loth est, au même titre qu'Un amour maladroit, un texte essentiellement narcissique. Comme Rachel, Hélène se racontera depuis sa tendre enfance jusqu'à la fin de sa liaison. De ce point de vue, ces histoires empruntent le même modèle: l'enfance, l'adolescence, l'âge adulte et l'expérience amoureuse. Seule diffère l'ordonnance du récit. Au récit linéaire analeptique d'Un amour maladroit se substitue dans la Femme de Loth, un récit, analeptique aussi, mais où sont intercalés l'un dans l'autre le passé éloigné (enfance, adolescence, jeunesse adulte) et le passé récent (la liaison) dans un découpage de petits chapitres, certains d'un seul paragraphe, numérotés de 1 à 240.

Mais au-delà de ces ressemblances superficielles, ces deux romans se rejoignent surtout au niveau de la forme de leur contenu. Dans chacun des romans, une femme, rétrospectivement, explicite la relation de son corps au monde, en fonction, non pas de choix délibérés, mais de déterminismes affectifs et physiologiques précis. Hélène et Rachel sont, non pas comme des soeurs, fussent-elles jumelles siamoises, mais comme deux masques derrière lesquels se profile, dirait-on, un seul personnage.

Comme Rachel, Hélène émigrera depuis la France vers le Canada pour s'installer à Montréal. Cependant, émigrée en 1938 avec ses parents, c'est à Montréal dans un couvent qu'elle complétera son éducation. Comme Rachel, Hélène n'est pas aimée de sa mère, mais non plus de son père; ceuxci considèrent leur fille comme une intruse. Et comme la mère de Rachel, la mère d'Hélène est très belle. Cependant dans le cas d'Hélène, le père aussi étant très beau, ses parents forment un couple uni ( $«$ Je n'ai jamais rencontré de couple mieux accordé15 $)$ ). Comme Rachel, c'est avec sa grand-mère qu'Hélène établit une certaine complicité, fondée dans les deux cas sur une ressemblance physique et morale. Bien qu'Hélène n'ait pas de petite soeur qui puisse lui servir, comme Élisabeth auprès de Rachel, de double positif, son amie intime, Danielle Guillaume, couventine aussi, assume auprès d'elle une fonction identique.

Là, cependant, où la ressemblance d'Hélène avec Rachel se manifeste avec le plus d'éclat, c'est des points de vue physique et psychologique. Car Hélène, la mal aimée de ses parents, souffre, elle aussi, d'avoir un corps ingrat: «... je me découvrais le fruit raté de leur commune maladresse ${ }^{16} \%$. Ce faible corps ( $\left\langle\mathrm{J}\right.$ 'étais frêle, malingre») ${ }^{17}$ finira par prendre des forces, mais il conservera sa gaucherie naturelle et, affreux petit canard, il restera, devenu grand, canard. Au plus grand dépit de son propriétaire:

J'ai rarement été «bien dans ma peau». Il y eut cette enfance, cette interminable adolescence où je m'agitais frénétiquement pour tenter une métamorphose. On parle beaucoup, aujourd'hui, du droit à la vie, à la santé, au bonheur. Mais le droit à la beauté? On me l'avait refusé. Révoltante injustice ${ }^{18}$. 
Tout le drame d'Hélène, comme d'ailleurs celui de Rachel, est résumé dans ce texte. Dans un monde où est exaltée la beauté physique, particulièrement la beauté du corps féminin, être privé de beauté, c'est être privé d'existence («Refoulée dans la non-existence $\left.\left.{ }^{19}\right\rangle\right)$. Surtout que dans ce même monde, l'ascension à la vie amoureuse pour la femme est fonction de la beauté ou non de son corps. Sans beauté donc, pas de vie amoureuse. Pas de vie amoureuse, c'est l'étiolement du corps et de l'être. Du moins, ce sont là les équations qu'établit le texte:

Hier, je ne me doutais de rien. Me voilà passée de vie à trépas. Le moi d'hier est mort. Depuis peu, il s'imaginait exister dans ton regard.

La tête vide. Le corps inutile. Ce corps soudain mis au rancart. Qu'en faire? Où le jeter, s'en débarrasser ${ }^{20}$ ?

Curieusement, ces dix ans que dura la liaison amoureuse sont présentés sous deux aspects, tous deux essentiellement négatifs: le moment de rupture, d'une part, et le caractère fugitif, intermittent de leur relation, d'autre part. Du temps de l'extase amoureuse, du temps de la jouissance, rien. Alors que le texte fait longuement état du corps en attente, puis du corps délaissé, abandonné, comme dans Un amour maladroit il fait le silence autour du corps qui jouit, qui exulte. Pourtant, n'est-ce pas là la grande préoccupation du roman? Â moins que ce corps, tour à tour malingre, disgracieux, gauche, détesté, délaissé, ne visât en fait à travers l'état extatique désiré, un autre état, plus stable celui-là, celui de la domesticité: le bonheur conjugal. ( J'ai passé des années à faire du lèche-vitrine aux étalages du bonheur conjugal'1»), un enfant ("Ce corps donc, ce corps à moi, après sa brève heure de gloire, on lui a refusé de s'épanouir en un vrai corps de femme, lourd et rond, múrissant comme un melon au soleil de septembre $\left.{ }^{22} \Downarrow\right)$.

Et c'est pourquoi le corps amoureux, le corps de «gloire», n'est qu'effleuré. Le texte, qui semble si préoccupé du corps, ne va jamais au-delà de sa surface, ne dépasse jamais sa couche épidermique. De beauté dans ce texte, il ne s'agit encore et toujours que de la beauté la plus visible, la plus voyante, tonitruante, mais de la beauté du corps secoué, traversé, transformé par le plaisir, jamais un mot. Sans aucun doute, cette censure est révélatrice du caractère fondamentalement médiateur du corps amoureux, mais elle met à jour, par ailleurs, l'essentielle incapacité de la part de l'héroïne de se définir depuis son être propre.

Privée d'un centre, dirait-on, privée d'une psyché suffisamment constituée pour se donner une vie autonome ( J'ai toujours été étonnée de voir mon ombre se profiler sur un mur, sur le sol'23") Hélène, comme Rachel eût espéré le faire avec Yves Dumont, vécut dix ans par procuration grâce à Pierre son amant. Pierre parti, le corps s'effondre, le moi menace de se 
désagréger et de sombrer dans la banalité. D'ailleurs, le sentiment de sa propre banalité n'est jamais très éloigné de l'esprit d'Hélène: «Je ne me suis jamais trouvée exceptionnelle, mais ignoblement banale ${ }^{24}$.»

Malheureusement, ce sentiment de banalité qu'entretient Hélène à son propre sujet, finit par être partagé par le lecteur. Ces jérémiades, de femme sans beauté et sans mari, répétées inlassablement du début jusqu'à la fin du roman finissent par fatiguer. Non que la souffrance d'une femme répudiée ne puisse soulever encore dans la vie réelle un sentiment de pitié. Mais comme sujet de roman... seule une écriture originale eût pu sauver une telle histoire du naufrage. On s'étonne d'avoir à lire un texte comme celui-ci, par exemple, où de chaque mot, de chaque phrase transpire la banalité du quotidien:

Je me défends encore. Seule, dédaignée, méprisée, laissée pour compte, je continue les recettes d'autrefois. Régime sévère, gant de crin, crèmes, pommades. Cheveux brossés, sourcils épilés. Et j'en passe ${ }^{25}$.

Bien sûr, ce texte souligne le drame du vieillissement du corps de la femme, mais cela tient du poncif. Et c'est sans doute le reproche le plus grave qu'on puisse formuler au sujet de ce roman, celui de n'avoir pu s'élever au-dessus des lieux communs. Le second reproche, c'est celui d'avoir tenté sur un nouveau canevas de brosser une vieille histoire. Le changement de décor, les modifications de détails, ne masquent pas l'identité des deux histoires. Il n'est pas jusqu'à la scène du miroir («Miroir, miroir, dis-moi que, pour lui, je suis celle qui compte ${ }^{26}$ )) ou ces velléités de suicide, qui chatouillent l'héroïne jusqu'à la toute fin de l'histoire, que la Femme de Loth emprunte d'Un amour maladroit. Entre ces deux romans, si semblables, Monique Bosco publia les Infusoires en $1965^{27}$.

Alain Deschênes, Jacques Tremblay, Carole Brown et Adolphe Cormier, quatre employés de la succursale à Montréal de l'agence de publicité américaine MacTavish décident, après avoir assisté à un congrès à Rome, de passer ensemble leurs vacances à Venise.

Renversant la direction que prennent les héroïnes des deux autres romans, ces quatre personnages laissent derrière eux Montréal, enveloppée dans la grisaille et la monotonie du travail, pour aborder Venise avec l'espoir que cette ville leur permettra enfin d'accéder au plein épanouissement de leur personne.

Sans intrigue, sans action pour ainsi dire, les Infusoires se bornent à présenter tour à tour au fil des chapitres chacun des personnages dont l'attitude psychologique et le comportement sont reliés dans un rapport de causalité, comme cela se passe dans Un amour maladroit et dans la Femme 
de Loth, à la situation qu'ils eurent, enfants, à subir. Bien que narré à la troisième personne, le récit n'est cependant assumé qu'exceptionnellement par l'instance narratrice, puisque chaque personnage à tour de rôle assume le point de vue du récit. Là où la focalisation narrative se fait par son entremise, le personnage, par un retour en arrière sur son enfance, est présenté au lecteur et "expliqué». On le voit, les techniques romanesques exploitées dans ce roman ne diffèrent que très peu de celles qu'a utilisées la romancière dans les deux autres romans. Des quatre personnages, est privilégiée Carole Brown, car c'est à elle qu'est attribuée la tâche en début de roman de détailler la situation des personnages dans leur lieu de travail. C'est à elle aussi qu'incombe la responsabilité de clore le récit. Enfin, c'est aussi à ce personnage que le roman consacre le plus grand nombre de pages.

À l'exception d'Alain Deschênes, qui regrette sa mère et qui déteste par conséquent Venise, tous les personnages anticipent, grâce à Venise et à son carnaval (symbole sans doute de son éternelle jeunesse) une naissance nouvelle, un nouveau point de départ. Mais toutes les attentes seront déçues. Â l'euphorie des premiers jours succédera la déception.

La relation d'actes manqués et d'occasions ratées révèle au lecteur les tares affectives dont sont affligés ces personnages et qui en font des soeurs et des frères de Rachel (Un amour maladroit) et d'Hélène (la Femme de Loth). Tous ensemble sont l'illustration vivante d'une fondamentale incapacité d'être. Au sujet d'Adolphe Cormier, au corps malingre et maladif, le narrateur tient les propos suivants (dont la ressemblance avec certains propos d'Anne Hébert est par ailleurs assez frappante):

Déjà le pas était franchi: il demeurait à l'écart, en marge, condamné à vivre en ce monde en n'en faisant point partie, spectateur aux yeux bandés, ermite et prisonnier à la fois ${ }^{28}$.

Cette caractérisation s'applique aussi bien aux trois autres personnages. À la surface, cette incapacité de vivre pleinement pourrait être imputable à l'aliénation collective du peuple québécois, mais une lecture attentive et une mise en relation avec les héroïnes des deux autres romans autorisent à ne lui attribuer que des causes d'ordre individuel. Certes, il n'en demeure pas moins vrai que ce roman, à cause de sa thématique, s'insère dans le courant de la littérature d'aliénation collective des années soixante. Toutefois, les causes d'aliénation dans ce roman ressortissent strictement au domaine de l'individu et non à celui de la collectivité.

À la fin du roman, Carole Brown, dont le corps (selon ce qui est révélé au cours du récit) ne put, malgré les efforts réitérés du fringant tombeur. Raymond Girard, s'ouvrir aux plaisirs charnels ni, par conséquent, accéder à la plénitude d'être («... Carole s'avouait son incapacité d'être ${ }^{29}$ )). tout à fait conforme évidemment au cliché de l'Anglais frigide, Carole Brown, 
donc, après Venise envisage de s'installer d'une façon à peu près définitive dans la monotonie et l'ennui d'une vie sans surprise. Comme Rachel et Hélène. Avec cette différence que, grâce à son séjour à Venise et à d'autres voyages similaires entrevus, Carole pourra désormais combattre l'insipidité de son existence par l'évasion dans le rêve: «Au lieu de l'ouvrir au monde, un autre monde lui était offert ${ }^{30} \%$. Cette fuite, à la limite touchant à la schizophrénie, n'est pas autre chose que le masque multiforme de la mort.

Ecrits par une femme, leurs personnages principaux, tous des femmes, les trois premiers romans de Monique Bosco que nous venons d'examiner seraient néanmoins, je le crains, jugés assez sévèrement si on avait à les juger à l'aune du féminisme d'aujourd'hui. Ils pourraient, cependant, aussi être proposés comme autant d'exemples de l'aliénation de la femme dans un monde où le phallus impose sa domination. Les romans de Bosco serviraient alors de contre-exemples. Quoi qu'il en soit, Rachel, Hélène et Carole obéissent toutes aux stéréotypes féminins de leur époque dans la mesure où elles attendent/ont attendu passivement l'épanouissement véritable de leur personne, tant physique que spirituelle, la totale révélation de leur être profond, le sésame-ouvre-toi de l'expérience amoureuse.

Mais l'infirmité charnelle ne frappe pas que les héroïnes; sauf une ou deux exceptions, tous les personnages de ces romans souffrent d'une manière ou d'une autre d'une incapacité de jouir. Dire que la chair est triste dans les romans de Monique Bosco tient de la litote. Et ce n'est en fait qu'une coïncidence qu'ils soient le reflet d'attitudes répandues dans la société québécoise de l'époque. Chacun des romans, par l'établissement d'une filiation causale précise, remonte aux causes premières dont pas une seule, sauf erreur, n'est de nature sociale. L'expérience individuelle, en dehors de l'exploitation de certains clichés culturels, ne rejoint jamais la collectivité, ses tabous et ses codes. Tout au plus celle-ci sert-elle de toile de fond. Les causes sont d'ordre personnel, familial ou situationnel.

En se plaçant d'un autre point de vue, celui de la production romanesque, il n'est peut être pas inutile de souligner que les trois premiers romans de Monique Bosco, avec tous ces personnages qui ne peuvent accéder à la vraie vie, qui ne font jamais autre chose que de vivoter, font mine de s'inscrire, ici, dans le courant de la littérature de l'aliénation collective et, en France, dans le grand courant littéraire de la période d'après-guerre où s'est développé un type de personnage qu'on a vite qualifié d'anti-héros et que préfigurent ou illustrent des personnages comme Meursault, Roquentin ou Molloy. Toutefois, grâce à la qualité de l'écriture et/ou grâce au discours ou philosophique ou social qui le plus souvent les accompagne ou qu'ils prédiquent, tous ces personnages transcendent leur propre banalité et accèdent paradoxalement à une certaine grandeur héroïque. Tel, cependant, n'est pas toujours le cas des personnages de Monique Bosco. Échappe toutefois en partie à ce jugement Rachel dans Un amour maladroit qui acquiert une 
certaine densité grâce au monde exceptionnel de la France occupée qui, en plus de servir de toile de fond à l'histoire relatée, agit aussi puissamment comme acteur.

1. Paris, Gallimard, 1961, 213 p.

2. Un amour maladroit, p. 11.

3. Ibid., p. 205.

4. Ibid., p. 9.

5. Ibid., p. 82.

6. Op. cit.

7. «Assise près d'eux, en une salle de théâtre ou de concert, je me dérobais à leurs regards. Je ne pouvais supporter que l'on m'examine. Une gêne intense me figeait sous l'oeil d'autrui. (...) Oppressée et malheureuse, je cachais mon visage entre mes mains, feignant de prendre une attitude absorbée. Je fondais sous leur regard; leur contact me répugnait. Quand ils tentaient de prendre ma main entre les leurs, il me fallait tout mon courage pour l'abandonner. Peu à peu, je sentais ma main se glacer. Ils n'insistaient pas.» (p. 137-138)

8. Ibid., p. 139-140.

9. Ibid., p. 205-206.

10. Ibid., p. 208.

11. Ibid., p. 207.

12 Paris/Montréal, Éditions Robert Laffont/Éditions HMH, 1970, 282 p.

13. La Femme de Loth, p. 14.

14: Ibid., p. 42.

15. Ibid., p. 25.

16. Ibid., p. 26.

17. Ibid., p. 28.

18. Ibid., p. 90.

19. Ibid., p. 136.

20. Ibid., p. 70.

21. Ibid., p. 240.

22. Ibid., p. 252.

23. Ibid., p. 147.

24. Ibid., p. 152.

25. Ibid., p. 92.

26. Ibid., p. 240

27. Montréal, Éditions HMH, 1965, 174 p.

28. Les Infusoires, p. 137.

29. Ibid., p. 130.

30. Ibid., p. 172. 